durch Botschafter Egbert von Graffennied und seine Gattin aufs liebenswürdigste empfangen. Bei alledem, sowie auf zwei kollektiv durchgeführten Exkursionen in den Schärengürtel und nach Uppsala, hatte man hinreichend Gelegenheit, Stockholm und seine Umgebung kennenzulernen, ein Stadtgebiet, das beim Neuling wie beim Wiederkommenden stets tiefe Eindrücke hinterläßt und zum guten Gelingen des 19. Geographenkongresses wohl seinen guten Teil beitrug.

\title{
DIE MENSCHLICHEN KÖRPERFORMGRUPPEN DER SCHWEIZERISCHEN REGIONEN
}

\section{$\mathrm{Zu}$ einem Markstein der Anthropologie}

Im Jahre 1946 erschien der erste Band der Anthropologia Helvetica, der auf Grund von sechsjährigen Untersuchungen (1927-1932) an 35500 schweizerischen Stellungspflichtigen ein Gesamtbild der Rassen- oder Körperformenstruktur der Schweiz zeichnete. (Geographica Helvetica 2, 1947, 275-277). Der Schöpfer und Initiant des gesamten Werkes, der damalige Ordinarius für Anthropologie der Universität Zürich, Prof. Dr. Otto Schlaginhaufen, erwarb sich damit das Verdienst, die erste umfassende Anthropologie unseres Landes geboten zu haben. Mit der Darstellung der Gesamtschweiz ließ er es indessen nicht bewenden. Es war für ihn vielmehr klar, daß bei deren landschaftlicher und völkischer Vielfalt auch eine regionale Untersuchung zu erfolgen habe. Ihre nicht minder interessanten und wertvollen Ergebnisse legte er nun vor kurzem in einem umfangreichen zweiten Band der «Anthropologia» nieder. ${ }^{*}$ ) Er trägt den Untertitel «Die Anthropologie der Kantone und der natürlichen Landschaften», womit bereits angedeutet wird, daß es SchlaginHACFEN darum ging, sowohl die Eigenart der menschlichen Körperformen der politischen Einheiten als auch der sogenannten Naturregionen herauszuarbeiten.

Der erste größere Teil des Werkes ist den Kantonen gewidmet, deren Darstellung jeweils durch zwei Tabellen eingeleitet wird. Sie fassen die 34 ermittelten metrischen und 5 deskriptiven Merkmale mit ihren statistischen Charakteristika bzw. prozentualen Frequenzen zusammen. An sie schließt sich eine Uebersicht über die natürlichen Gebiete - für deren Abgrenzung zur Hauptsache die "Geographie der Schweiz" von J. FRÜH (1930-1945) maßgebend war - , in welche die Kantone aufgeteilt worden sind. Sie enthält jeweils eine Liste der Gemeinden, von welchen Stellungspflichtige untersucht werden konnten, wobei auch die Zahl der Untersuchten angegeben ist. Es wird so jedem Lokalforscher ermöglicht, für sein Gebiet die anthropologische Eigenart zu bestimmen. Den Hauptteil dieser Kapitel bestreitet die Beschreibung der Verteilung der einzelnen Merkmale innerhalb der Kantone; sie wird durch eine Tabelle zu lesen erleichtert, welche wiederum die statistischen Charakteristika bzw. die prozentualen Frequenzen des Merkmals in den einzelnen Gebieten enthält. Diese Tabelle bildet mit den 63 in einem besondern Atlas zusammengefaßten kartographischen Darstellungen die Grundlage für die Beschreibung der räumlichen bzw. landschaftlichen Verteilung der Merkmale. An solchen wurden berücksichtigt: die Körpergröße, die Körperhöhe im Sitzen, die relative Körperhöhe im Sitzen, die Beinlänge, die Spannweite der Arme, die relative Spannweite, die größte und die relative Kopflänge, die größte und die relative Kopfbreite, der Längenbreiten-Index des Kopfes, die kleinste Stirnbreite, der Transversale Fronto-parietal-Index, die morphologische und die relative Gesichtshöhe, die absolute und relative Jochbogenbreite, der morphologische Gesichts-Index, die physiognomische Obergesichtshöhe, der physiognomische Obergesichts-Index, die Unterkieferhöhe, die Unterkieferwinkelbreite, der Index fronto zygomaticus, der Index mandibulo-zygomaticus, der transversale Kephalo-facial-Index, die Breite zwischen den innern und den äußern Augenwinkeln, der Augendistanz-Index, die Höhe und die Breite der Nase, der Nasen-Index, der transversale und der sagittale Naso-facial-Index, der Nasenbreiten-GesichtshöhenIndex, das Profil der Nase und des Hinterhaupts, die Form der Haare sowie die Farbe der Augen und Haare, woraus sowohl die Differenziertheit der Untersuchung wie ihre Schwierigkeiten ersichtlich werden. Auf die Behandlung dieser Einzelmerkmale und Indizes folgt diejenige der Merkmalskombinationen, die erst die Rassen- bzw. Körperformenstruktur eines Gebietes richtig zu beurteilen erlauben. Sie mußten raumeshalber stark gekürzt werden, so daß die beiden für jeden Kanton erstellten Tabellen sich auf die Sechserkombination Körpergröße, Längenbreiten-Index des Kopfes, morphologischer Gesichts-Index, Nasen-Index, Augen- und Haarfarbe beschränken. In der ersten der dieser gewidmeten Tabellen sind die in jedem Kanton am häufigsten auftretenden Verbindungen nach der Häufigkeit ihres Vorkommens aufgeführt. Die Zahl der berücksichtigten Nummern hängt jeweils von der Größe

*) Anthropologia Helvetica. Ergebnisse anthropologischer Untersuchungen an schweizerischen Stellungspflichtigen II. A. Textband 708 Seiten (970 Tabellen). B. Atlas. Von Otto Schlaginhaufen. Zürich 1959. Art. Institut Orell Füssli AG. 
des Kantons bzw. von der Zahl der untersuchten Kantonsbürger ab. In Kanton Luzern wurde sie z. B. auf 21, im Kanton Bern auf 50 festgelegt. Die zweite Tabelle registriert diejenigen Verbindungen der Sechserkombination, welche in den einzelnen natürlichen Regionen des Kantons am häufigsten vorkommen. In beiden Tabellen ist zudem angegeben, welchen schweizerischen Ordnungsnummern die häufigsten Verbindungen im Kanton bzw. Gebiet entsprechen.

Um einen Begriff von der Reichhaltigkeit der Darstellung zu geben, die natürlich nur ein eingehendes Studium ausschöpfen kann, seien einige Hinweise auf die Anthropologie des Kantons Zürich geboten, in welchem 3468 Bürger gemessen worden waren. Seine regionale Gliederung umfaßt 17 Gebiete: Zürich-Stadt, Limmattal, Knonaueramt und Reppischtal, Sihltal, linkes und rechtes Zürichseeufer, Greifenseegebiet, Pfäffikerseegebiet mit Kempttal, Jonatal, oberes Tösstal, Winterthur, Eulachtal, Thur-Rheingebiet, Tössmündungsgebiet, Rafzerfeld, Glattal sowie Furt- und Wehntal. Mit der Körpergröße von im Mittel $169,53 \mathrm{~cm}$ nimmt der Kanton den 21. Rang ein und sie erhebt sich rund $1 \mathrm{~cm}$ über das schweizerische Mittel, die Zürcher erscheinen somit als relativ "große» Leute, wobei sich die Bewohner der Städte Zürich $(170,7 \mathrm{~cm})$ und Winterhur $(170,36 \mathrm{~cm})$ inkl. das Limmattal $(170,46 \mathrm{~cm})$ deutlich von den ländlichen Gebieten (unter $170 \mathrm{~cm}$ ) abheben. Für die größte Kopflänge wurden im Mittel 289,3 mm gemessen, wonach Zürich etwas über demjenigen der Schweiz stebt, aber auch in dieser Hinsicht den 15. Rang einnimmt und wiederum die Stadt mit 190,69 mm über die Landgebiete dominiert (Winterthur 190,24, Limmattal 190,2 mm). Den gleichen Rang hält mit 81,42 der Kanton hinsichtlich des Längenbreiten-Index des Kopfes inne, wobei im ganzen kleine Werte bei einer regionalen Variationsbreite von 80,61 (Stadt Zürich) bis 82,61 (Oberes Tösstal) fehlen, die Zürcher sich also wie die übrigen Schweizer (Mittel 81,31) als Breitköpfe oder Brachykephale erweisen. (Die breitköpfigsten Schweizer sind die Innerrhoder Appenzeller mit den Tessinern und Wallisern, die am wenigsten brachykephalen die Westschweizer: Genfer, Waadtländer, Neuenburger und die Basler). Hinsichtlich der Haarform gehört der Kanton Zürich zum Gebiet der Schlichthaarigen, die 78,0\% aller Gemessenen (Schweiz 80,14\%) ausmachen, womit er an 5 . Stelle aller Stände steht. Die regionalen Unterschiede sind gering. Die Farbe der Augen variiert relativ stark, wobei die melierten mit 33,85\% überwiegen, wenn auch nicht in allen Gebieten (vornehmlich braunäugig sind die Bewohner der Stadt Zürich, des Knonauer Amtes, sowie des Pfäffikersee- und Jonagebietes). Bei den Farben der Haupthaare dominiert braun mit $57,28 \%$, doch liegt der Kanton Zürich in dieser Beziehung deutlich unter dem schweizerischen Mittel $(63,32 \%)$, wobei regional Schwankungen von $51,28 \%$ (Thur-Rheingebiet) bis $67,48 \%$ (Jonatal) auftreten. Im übrigen erweist sich der Kanton als ein Gebiet relativ häufiger Blondhaarigkeit (insbesondere mit verhältnismäßig zahlreichen Hellblonden : 3,3\%). Bezüglich der sogenannten Sechserkombination schließlich stimmt er weitgehend mit der Gesamtschweiz überein, d. h. die Verbindung von großen, mesokephalen, leptoprosopen, leptorrhinen Typen bei melierten Augen und braunen Haaren herrscht mit $1,88 \%$ deutlich vor, doch haben bei einer Gesamtzahl von 791 Verbindungen eine größere Zahl analoge Prozente (1-1,38). Mit diesen wenigen Angaben ist die anthropologische Charakteristik des Kantons Zürich und seiner Regionen nur angedeutet, jedoch belegen sie zweifellos die außerordentliche Reichhaltigkeit und Gründlichkeit des Gesamtwerkes, die bisher von wenigen ähnlichen Unternehmungen erreicht wurden.

Da im Kapitel Kantone die "natürlichen Landschaften» bereits weitgehend diskutiert sind, erübrigte sich im zweiten Hauptteil a Die Anthropologie der natürlichen Landschaften der Schweiz * eine nochmalige detaillierte Darstellung. Diese wurde deshalb mit Recht in Form einer Zusammenfassung der wesentlichen Ergebnisse gehalten, wobei hier lediglich auf einige wenige Punkte hingewiesen werden kann, die den besondern Wert gerade einer solchen Uebersicht beleuchten sollen. Die kantonsweise Beschreibung hatte z. B. erkennen lassen, daß die kleineren Körpergrößen (bis $167 \mathrm{~cm}$ ) in vier Zentren: St. Gallen-Appenzell, Schwyz-Uri-Obwalden und Luzern, Tessin sowie Freiburg angeordnet sind. Die Karte der kleinern Regionen ergibt, daß diese Zentren nicht mehr so eng umgrenzt sind und neue Zusammenhänge mit Nachbargebieten aufweisen sowie, daß außerhalb derselben sekundäre Zentren auftreten, z. B. im Thurgau, in Tösstal, auf dem Kerenzerberg usw. Bemerkenswert ist weiterhin in diesem Zussmmenhang, daß die größern Städte sich als Gebiete größerer Körperlänge von ihren Umgebungen abheben, am auffälligsten St. Gallen, an dessen Grenzen Bezirke kleinen, unter- und übermittelgroßen Wuchses anstoßen, wobei die Städte Frauenfeld, Schaffhausen, Winterthur, Zürich, Zug, Luzern, Aarau, Olten, Basel, Solothurn und Bern Enklaven übermittelgroßen Wuchses darstellen. In der räumlichen Verteilung des LängenbreitenIndex des Kopfes treten die Ausläufer des mesokephalen Blockes, der die Kantone Freiburg, Neuenburg, Waadt und Genf umfaßt, schärfer hervor, indem "Wachstumsspitzen» Richtung Simmental, Bern, Thun usw. weisen. Der weitaus größte Teil der Schweiz ist jedoch von Feldern des Index 81 eingenommen, die von den Freibergen bis ans Ostende des Prättigaus, nordsüdlich von Koblenz bis Adelboden reichen. Hinsichtlich der Haupthaarfarbe schließlich ist kennzeichnend, daß zahlreiche Gebiete bestehen, in welchen *Hellblond * nicht gemeldet wurde, während *Blond , mit Ausnahme der West- und Südschweiz, in einem Block größerer Häufigkeiten auftritt, «Braun» umgekehrt vor allem die ersteren Gebiete charakterisiert.

Mancher Geograph, Volkskundler, Soziologe oder auch Anthropologe wird im Blick auf das imponierende Gesamtwerk bedauern, daß dieses sich auf die *Körperformgruppen* beschränkt, die psychophysische Einheit des Menschen unberücksichtigt ließ, welcher eine umfassende Anthropologie 
ihrem Namen gemäß doch zugleich gewidmet sein sollte. Der Verzicht auf diesen Versuch kann aber dem nicht unklar sein, welcher die Schwierigkeiten zu beurteilen vermag, die, schon in der Konzeption des Begriffs liegend, einer methodisch-arbeitstechnischen Erfassung entgegenstehen. Es ist deshalb im Gegenteil zu bewundern, daß entgegen vielen andern Fachgenossen der Autor der Anthropologia Helvetica, sich in der Untersuchung der physischen Anthropologie beschied.

Wer die vorbildliche Arbeitsweise Professor Schlaginhaufens kennen zu lernen Gelegenheit gewann, war sich klar, daß er sein Werk keineswegs allein auf die eigenen grundlegenden Untersuchungen abstellte, sondern auch die vordem durchgeführten Arbeiten sorgfältig berücksichtigte, wobei er erfreulicherweise auf eine große $Z$ ahl von Studien seiner eigenen Schüler zurückgreifen konnte. Die Anthropologia Helvetica ist daher auch zugleich als retrospektive Bibliographie der anthropologischen Literatur der Schweiz wertvoll, auf welche auch aus diesen Gründen immer wieder gefußt werden muß.

Der Autor dankt in seinem Vorwort einer größeren Zahl von Helfern, unter welchen hier das Eidgenössische Militärdepartement, die Julius Klaus-Stiffung und der Schweizerische Nationalfonds zur Förderung der wissenschaftlichen Forschung, nicht zuletzt seine Sekretärin Frl. Helene Bl.EuleR genannt seien, welch letztere das Werk von seinen ersten Anfängen an mit großem Verständnis und unermüdlicher Pflichttreue begleitet hat. Mitarbeiter, Verlag Art. Institut Orell Füssli AG., welcher auch dem zweiten Teil der Anthropologia Helvetica eine würdige Form verlieh, und vor allem der Autor selbst sind zu diesem Markstein der schweizerischen Anthropologie nicht nur, sondern der Menschenforschung überhaupt, zu beglückwünschen. Es liegt in ihm eine wissenschaftliche Leistung vor, die über das Fach hinaus alle Disziplinen vom Menschen dauernd befruchten wird.

E. WINKLER

\section{NEUERE MORPHOLOGISCHE STUDIEN}

Im Jahre 1958 erschien zum sechzigsten Geburtstag von Hans Spreitzer, Ordinarius für physische Geographie an der Universität Wien eine Festschrift, die Wert ist, daß sie auch an dieser Stelle gewürdigt wird. Eingangs wird, wie es sich gehört, durch G. BARTsCH das wissenschaftliche Werk des Jubilars eingehend gewürdigt. Neben siedlungsgeographischen, alpwirtschaftlichen und länderkundlichen Fragen haben H. SPREITZER hauptsächlich geomorphologische Probleme intensiv beschäftigt; eine Reihe wertvoller, auf gründlichster Beobachtung fußender Publikationen zeugt von seinem sehr erfolgreichen Schaffen. Reisen und längere Aufenthalte machten ihn, von seinem Heimatland abgesehen, mit Niedersachsen, Böhmen, Rußland und Kleinasien vertraut. Als Zeichen der Anerkennung und des Dankes ist dem hochgeschätzten Forscher und akademischen Lehrer die vorliegende Festschrift auf den Geburtstagstisch gelegt worden. Sie enthält 18 wissenschaftliche Arbeiten, die von einer einzigen Ausnahme abgesehen, physisch-geographische und im besondern morphologische Themen beschlagen. Der größere Teil davon hat Gebiete des alpinen Raumes zum Gegenstand.

A. Winkler v. Hermaden unternimmt die schwierige Aufgabe, die großen Züge im Formenbild der östlichen Alpen in ihrer engen Zusammengehörigkeit und genetischen Einheitlichkeit aufzuzeigen und zu deuten. Ein prägnantes und fesselndes Gesamtbild der geomorphologischen Entwicklung dieses alpinen Raumes ersteht vor unserem geistigen Auge. Der Beitrag von TH. PIPPAN befaßt sich vor allem mit dem Einfluß tektonisch-petrographischer Strukturen auf den Formenschatz des Stubachtales in den Hohen Tauern. Dabei wird namentlich den Problemen der Bildung der Stufen, die sowohl das Tal als auch die Gipfelflur in ihrer stockwerkartigen Gliederung auszeichnen, die Aufmerksamkeit zugewandt. Diese Bildung ist teils als das Ergebnis einer phasenhaften Heraushebung des Gebirgskörpers, teils als das eiszeitlicher Glazialerosion anzusehen. Besonderes Interesse erweckt sodann der Bericht von L. WeINBERGER über den Bau und die Bildung des Ibmer Moos-Beckens im Gebiet der Salzach (Oberösterreich) mit seinen verschiedenaltrigen Moränen, verschiedenartigen Toteisformen, wie Söllen, Randterrassen, Kames und Osern und einstigen Eisstauseen. Mit Hilfe von Bohrungen konnte die Sohle dieses Tertiärbeckens ermittelt werden. Aus den Zahlen, geht hervor, da $\beta$ die Eintiefung durch den ehemaligen Gletscher nicht besonders bemerkenswert war. Ein Aufsatz von J. ZörL beschäftigt sich eingehend mit den Problemen der Karsthydrographie unter besonderer Berücksichtigung der Frage des Erosionsniveaus. Er gibt nicht nur einen Überblick über den derzeitigen Stand der Karstforschung, sondern legt neue Untersuchungsergebnisse vor, die der Autor in verschiedenen alpinen Karstgebirgen gewonnen hat. $\mathrm{H}$. КоHL, dessen Beitrag uns in die südwestliche Traun-Enns-Platte führt, vermochte deren Blockablagerungen auf Grund der petrographischen, stratigraphischen und pedologischen Eigenschaften einwandfrei als Altmoränen, wahrscheinlich aus der Günzeiszeit stammend, zu identifizieren. J. MATZNETTER äußert sich über Wesen und Begriff des alpinen Elementarereignisses, worunter er bestimmte Vorgänge, wie Erdbeben, Hochwasser, Bergstürze usw., versteht, und S. MoRAWETz über die Tobel östlich von Graz, deren es über hundert gibt, die sich auf Grund ihrer Form zu zwölf Typen zusammenstellen 